\title{
Análisis de las causas de deterioro en sistemas de almacenamiento magnético de datos
}

\section{Failure Analysis of Storage Data Magnetic Systems}

\author{
Ortiz-Prado A. \\ Laboratorios de Ingeniería Mecánica Ing. Alberto Camacho Sánchez \\ Facultad de Ingeniería, Universidad Nacional Autónoma de México \\ E-mail:armandoo@unam.mx \\ Schouwenaars-Franssens R. \\ Laboratorios de Ingeniería Mecánica Ing. Alberto Camacho Sánchez \\ Facultad de Ingeniería, Universidad Nacional Autónoma de México \\ E-mail:raf_shouwenaars@yahoo.com \\ Jacobo-Armendáriz V.H. \\ Laboratorios de Ingeniería Mecánica Ing. Alberto Camacho Sánchez \\ Facultad de Ingeniería, Universidad Nacional Autónoma de México \\ E-mail:vjacobo@dgapa.unam.mx \\ Sánchez-Pérez F. \\ Laboratorios de Ingeniería Mecánica Ing. Alberto Camacho Sánchez \\ Facultad de Ingeniería, Universidad Nacional Autónoma de México \\ E-mail:fco.san@comunidad.unam.mx
}

(Recibido: agosto de 2009; aceptado: abril de 2010)

\section{Resumen}

En este trabajo se presentan los resultados obtenidos de la inspección de 198 unidades de almacenamiento magnético de datos (discos duros), que estuvieron en servicio en nueve diferentes regiones climáticas características para la República Mexicana. Los resultados permiten definir las tendencias en cuanto al tipo de falla y los factores que inciden en éstas. A su vez, se han analizado las causas que conducen a la falla de tipo mecánico y las debidas a deterioro por corrosión atmosférica.

Con base en los resultados obtenidos de muestras de campo, se demuestra que la falla en discos duros es fundamentalmente por efectos mecánicos. El deterioro por efectos ambientales se observó en cabezas de lectura-escritura, circuitos integrados, circuitos impresos y en algunos de los componentes electrónicos de la tarjeta controladora de dicho dispositivo, pero no en las superficies de almacenamiento magnético. Es por tanto que se puede descartar a la corrosión en la superficie del disco como un tipo de deterioro que afecte directamente el funcionamiento de dichos dispositivos por efecto del medio ambiente. Para evitar cualquier inconveniente en el sistema de almacenamiento magnético de datos es necesario garantizar hermeticidad en el sistema.

Descriptores: disco duro, corrosión atmosférica, corrosión en equipo electrónico. 
DOI: http://dx.doi.org/10.22201/fi.25940732e.2010.11n4.037

Análisis de las causas de deterioro en sistemas de almacenamiento magnético de datos

\begin{abstract}
This paper shows the conclusions about the corrosion mechanics in storage data magnetic systems (hard disk). It was done from the inspection of 198 units that were in service in nine different climatic regions characteristic for Mexico. The results allow to define trends about the failure forms and the factors that affect them. In turn, this study has analyzed the causes that led to mechanical failure and those due to deterioration by atmospheric corrosion. On the basis of the results obtained from the field sampling, demonstrates that the hard disk failure is fundamentally by mechanical effects. The deterioration by environmental effects were found in read-write heads, integrated circuits, printed circuit boards and in some of the electronic components of the controller card of the device, but not in magnetic storage surfaces. Therefore, you can discard corrosion on the surface of the disk as the main kind of failure due to environmental deterioration. To avoid any inconvenience in the magnetic data storage system it is necessary to ensure sealing of the system.
\end{abstract}

Keywords: Hard disk, atmospheric corrosion, corrosion in electronic equipment.

\section{Introducción}

Los sistemas de almacenamiento magnético de datos (SAMD) al igual que los dispositivos electrónicos, son susceptibles a deteriorarse, debido a efectos eléctricos, magnéticos, mecánicos y químicos. Por los materiales empleados se ha considerado que la superficie de almacenamiento magnético puede presentar deterioro por corrosión atmosférica (Ortiz, 2004).

La corrosión, de partes o componentes de dispositivos electrónicos y de los SAMD, puede producir un amplio espectro de consecuencias que van desde ruido en la señal, fallas intermitentes y pérdida de información; hasta la incapacidad total del equipo para seguir operando. Si bien, algunas de estas fallas, sobre todo en el caso de las de tipo intermitente, no pueden ser catalogadas estrictamente como corrosión, sí se producen por efecto de precursores de ésta, como es el caso de la generación de cortocircuitos entre pistas por la presencia de humedad, o fallas en elementos magnéticos por polvo o partículas producto de procesos corrosivos (Gouda et al., 1989).

El deterioro por corrosión de componentes de equipo electrónico es más crítica que la corrosión de otros sistemas, ya que no se demanda una sensible pérdida de masa como en los materiales estructurales, para que se presenten inconvenientes en la operación del sistema (Ortiz, 2004). Esto se debe a que la mínima degradación o deterioro afecta las propiedades eléctricas ó magnéticas del dispositivo (conductividad, magnetización, etc.), y por consecuencia, su desempeño.

Desde los años 60s de la pasada centuria, diversos autores (Guttenplan, 1987; White et al., 1987) han considerado que el equipo y dispositivos eléctricos, electrónicos y magnéticos pueden presentar fallas inducidas por corrosión, por tal motivo, es conveniente orientar el análisis de acuerdo a las características particulares de los diferentes elementos que se pueden encontrar en los dispositivos electrónicos para un análisis más preciso. En este sentido, se ha propuesto (Ortiz, 2004) clasificar a los dispositivos con base en sus dimensiones, características y aplicación de la siguiente forma:

a) Dispositivos de microelectrónica (circuitos impresos, circuitos integrados).

b) Dispositivos de macroelectrónica. Interruptores, conectores, resistencias, capacitores, pilas, etc.

c) Dispositivos de almacenamiento magnético de datos (SAMD).

Cerrud et al. (2003) y Ortiz (2004) reportan el efecto que las variables ambientales tienen en el deterioro que, por corrosión, sufren dispositivos tales como: conectores, interruptores o tarjetas.

Es por consecuencia que en este artículo se reporta la influencia que el medio ambiente tiene en el deterioro de los SAMD.

Los SAMD (figura 1) son dispositivos de almacenamiento masivo de información, si estos sufren algún daño o mal funcionamiento sería catastrófico por las pérdidas que se tendrían con respecto a la información contenida, por ello, es importante analizar el por qué de su deterioro y cuáles son las causas que provocan la producción de fallas.

Estrictamente hablando, el elemento principal de los SAMD es el plato, siendo el elemento de almacenaje de información y la correspondiente cabeza de lectura-escritura. En todos los casos, éstos se encuentran al 
interior de una carcasa, la cual pretende ser totalmente hermética. La naturaleza del plato consta de diferentes capas de sustratos y materiales base (figuras $2 \mathrm{a}$ y $2 \mathrm{~b}$ ), los cuales se han ido modificando para obtener mejores prestaciones, buscando entre otros objetivos disminuir el desgaste a través de recubrimientos.

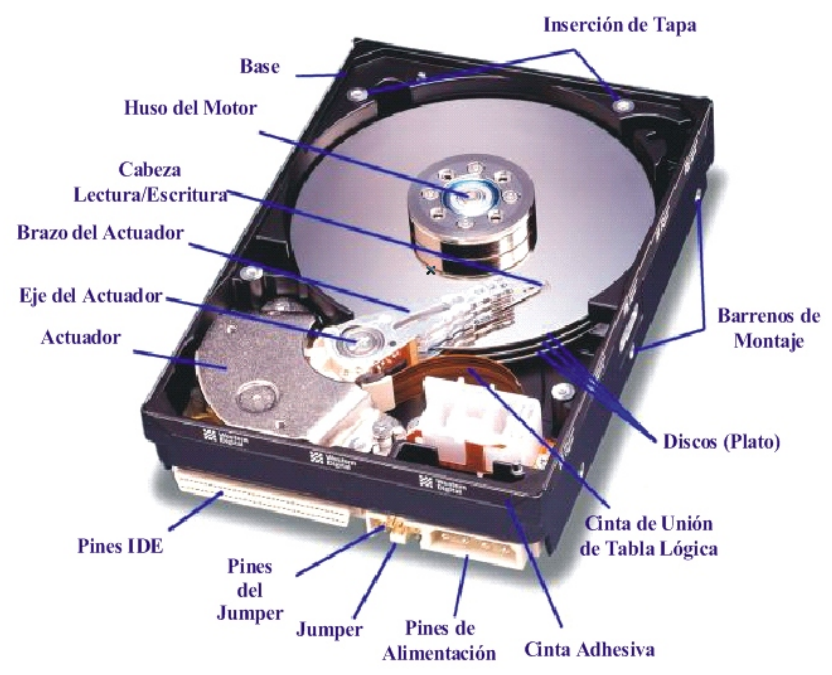

Figura 1. Principales elementos que componen un SAMD (Barrios et al., 2005)

a)

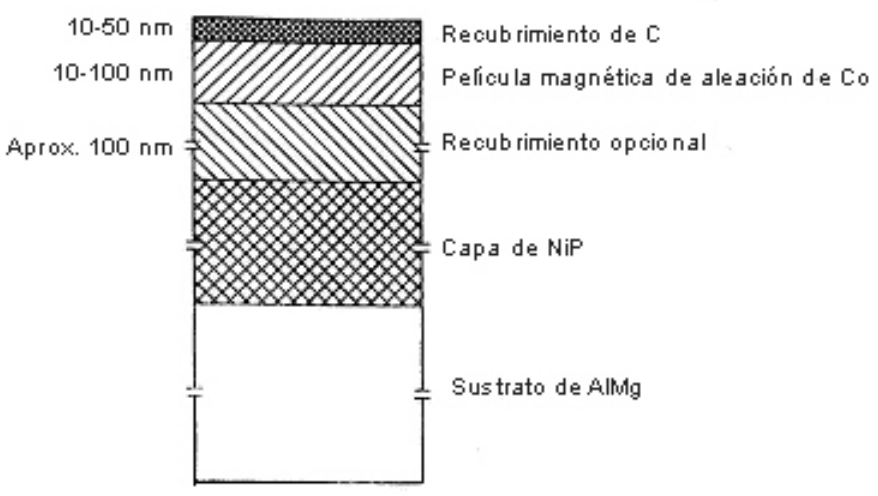

Fundamentalmente, la inquietud por el estudio del fenómeno de la corrosión atmosférica (CA) en SAMD, se basa en lo reportado en la literatura de Marcus (2002), en donde se menciona la existencia del deterioro por CA en la superficie del plato del disco duro (figura 3), la cual desde el punto de vista de la termodinámica es factible, siempre y cuando exista la presencia de humedad. La susceptibilidad al deterioro por corrosión del recubrimiento magnético del disco duro se ha reducido en los últimos años (Marcus, 2002), esto con base en los resultados de los estudios de polarización, donde se ha comparado el comportamiento del recubrimiento de cobalto con modificaciones del mismo. De las curvas de polarización (figura 4) se desprende que la densidad de corriente se reduce en varios órdenes de magnitud al emplear un recubrimiento $\mathrm{CoCr}$ en lugar del base cobalto o del CoP.

Conscientes de la problemática relacionada con la composición de la superficie magnética, queda pendiente el análisis de la frecuencia con la cual este tipo de fenómenos representan la causa de falla del disco, tomando en consideración las condiciones de exposición en las que se encuentra el dispositivo. Es por tal motivo que se procedió al diagnóstico y evaluación de dispositivos provenientes de exposición en campo bajo diferentes condiciones climáticas. Lo anterior, permite la determinación de las causas de deterioro y su frecuencia, para así instrumentar las acciones que permitan una mayor protección y duración del SAMD.

b)

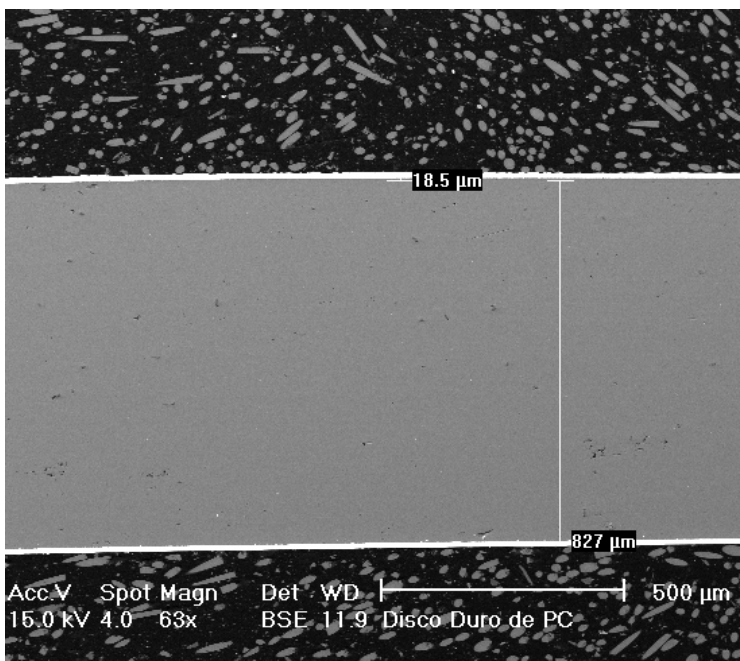

Figura 2. a) Composición del Plato del Disco Duro (Marcus, 2002). b) Sección Transversal, se observa que el plato está formado por un elemento base de AlMg de aproximadamente $820 \mu$ de espesor, sobre el cual a ambos lados se deposita la aleación Ni-P (zona más clara), dado que la capa de cobalto es muy delgada (del orden de $0.1 \mu$ ) y su densidad es similar a la del níquel, tiende a confundirse con ésta (Ortiz, 2004) 


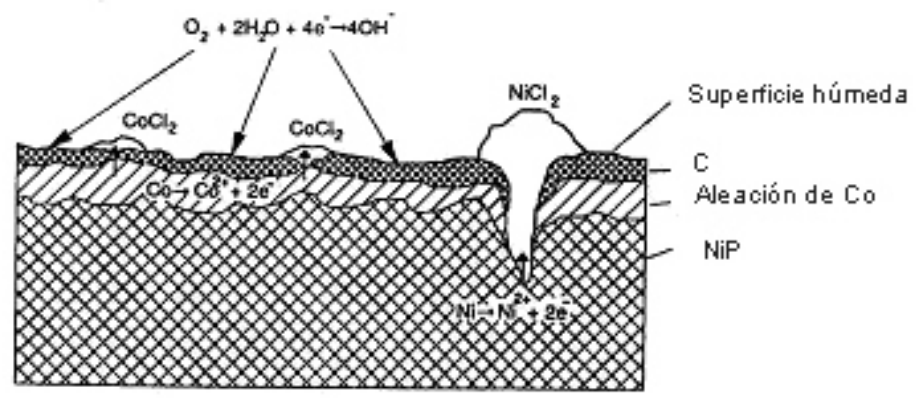

Figura 3. Esquema de corrosión en la estructura del disco en presencia de cloruros (Marcus, 2002)

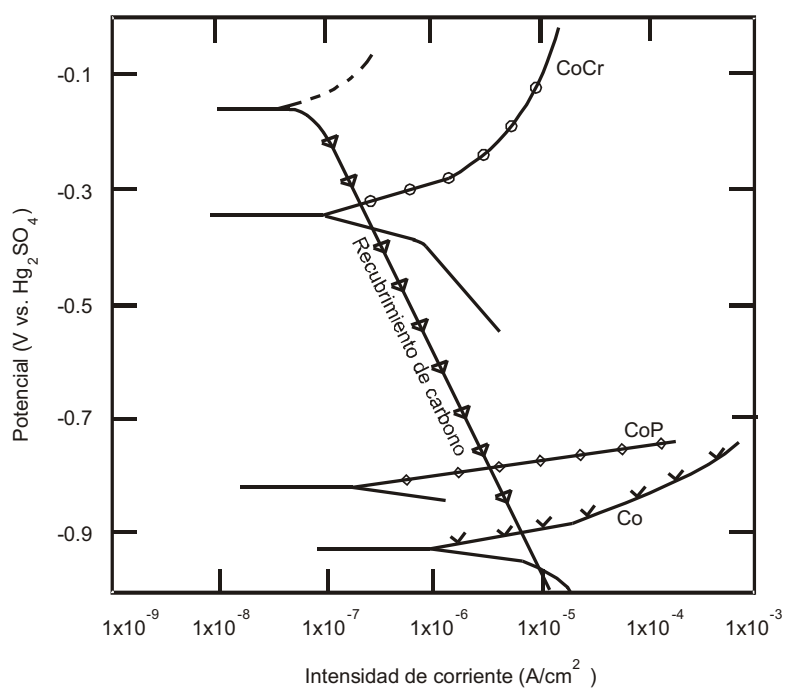

Figura 4. Curvas de polarización para recubrimientos magnéticos empleados en el disco duro, en combinación con la capa superficial de carbono.

El empleo de aleaciones $\mathrm{CoCr}$ reduce sensiblemente la densidad de corriente (Marcus, 2002)

\section{Metodología}

Para poder llevar a cabo esta evaluación se plantea una metodología de inspección (figura 5) con la finalidad de tener un procedimiento para la evaluación y análisis de aquellos especímenes que han presentado falla o en los cuales se han encontrado evidencias de posible deterioro por corrosión. De acuerdo a la metodología planteada se procede a la inspección en 198 muestras (figura 6).

\section{Tipos de fallas presentes en SAMD}

Como parte inicial de la evaluación se realiza un diagnóstico mediante paquetería especializada al dispositivo. Para

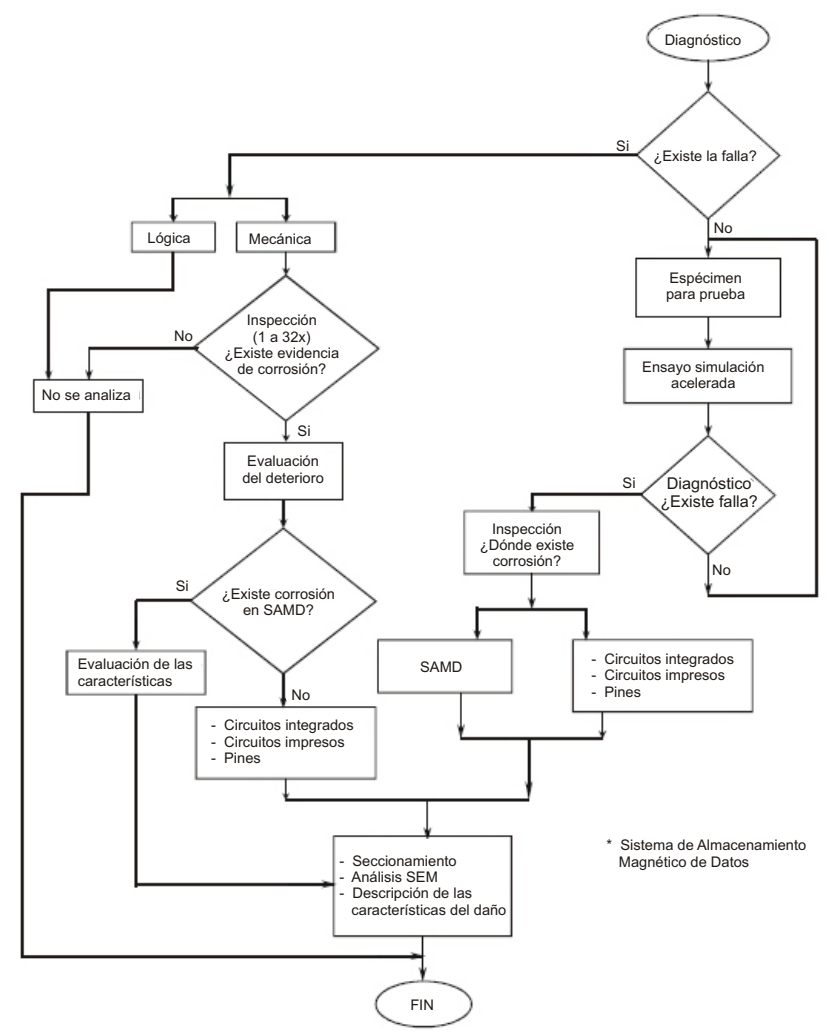

Figura 5. Metodología para evaluación del deterioro en SAMD (Ortiz et al., 2005)

facilitar el estudio, las causas de falla se han clasificado en cuatro grupos (Barrios et al., 2005):
a) Falla electrónica.
b) Falla mecánica.
c) Falla lógica.
d) Falla por corrosión. 


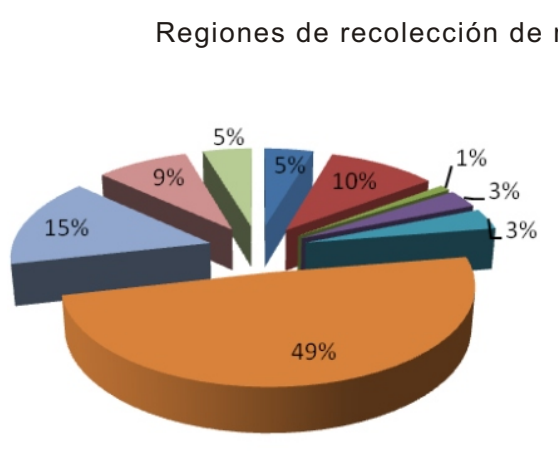

\author{
" Monterrey, N.L. \\ - Torreon, Coah. \\ Zacatecas, Zac. \\ - Aguascalientes, Ags \\ n San Luis Potosí, SLP \\ Cd México \\ - Guadalajara Jal. \\ - Villahermosa Tab. \\ mérida, Yuc.
}

Figura 6. Distribución de las

regiones de recolección de $S A M D$

La hipótesis original considera que la falla por corrosión es, si no la más importante, una de las principales que llevaban al mal funcionamiento de los SAMD.

\section{Desarrollo del estudio}

El resultado del diagnóstico inicial, de acuerdo con la metodología planteada, se presenta en la figura 7.

Deterioro por corrosión atmosférica en componentes electrónicos de SAMD
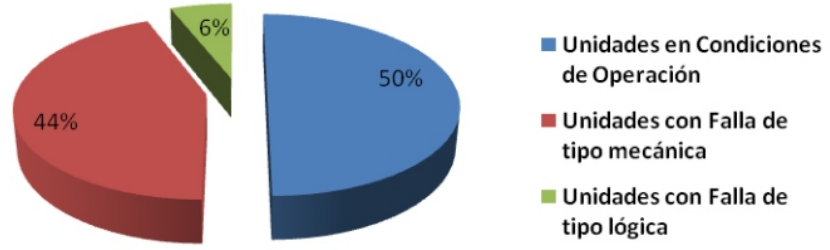

- Unidades en Condiciones de Operación

= Unidades con Falla de tipo mecánica

- Unidades con Falla de tipo lógica

Figura 7. Diagnóstico realizado a especímenes de estudio. En este caso las fallas debidas a problemas en la electrónica del sistema se han agrupado con las mecánicas

De los resultados obtenidos, es de resaltar que el $44 \%$ corresponde a falla mecánica. En la mayor parte de las unidades inspeccionadas existe deterioro en la superficie del disco, debido a la colisión con el cabezal de lectura - escritura (L/E), o bien, por la adhesión entre estos elementos (figura 8).

La frecuencia con la que se presentan los diferentes fenómenos involucrados en una falla mecánica se muestra en la figura 9. Dentro de las "otras causas" se encuentran inconvenientes, producto del mal funcionamiento de algún elemento electrónico, o bien, por factores como la falta de hermeticidad de la carcasa, lo cual se traduce en presencia de humedad y de partículas, entre otros.

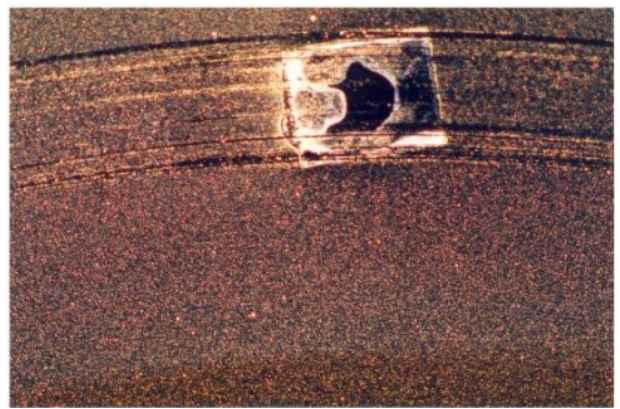

Figura 8. Adhesión del cabezal de L/E sobre la superficie del plato, debido a la presencia de humedad

Factores que conllevan a una falla de tipo mecánica

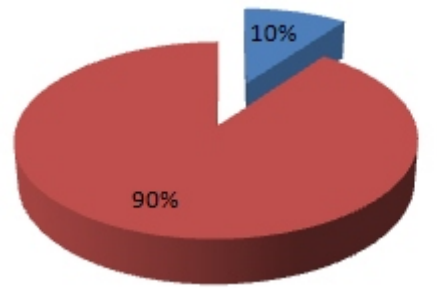

\section{- Otras Causas \\ - Colisión entre cabezal y plato}

Figura 9. Las fallas mecánicas del SAMD tienen, en gran medida, su origen en el impacto entre cabezal y plato, situación que favorece el desprendimiento del recubrimiento de la superficie del plato

\section{Resultados de la inspección}

Aquellas unidades que presentan falla mecánica se inspeccionaron a bajos aumentos y por microscopia electrónica de barrido (MEB).

En la mayoría de los casos, se observó acumulación de polvo sobre componentes electrónicos, así como cambio de tonalidad en pines de conexión y pistas de circuitos impresos.

Cabe mencionar que en muestras provenientes de la costa, se puede apreciar la formación de sulfuros en pines IDE.

En lo que corresponde a la inspección de la superficie del disco, se aprecia el desgaste del mismo por efectos mecánicos (figuras 10 y 11), esto tiene su origen en la holgura existente entre la cabeza de lectura-escritura con la superficie de almacenamiento magnético, que es cada día menor esto en función del incremento en la capacidad de almacenamiento. 

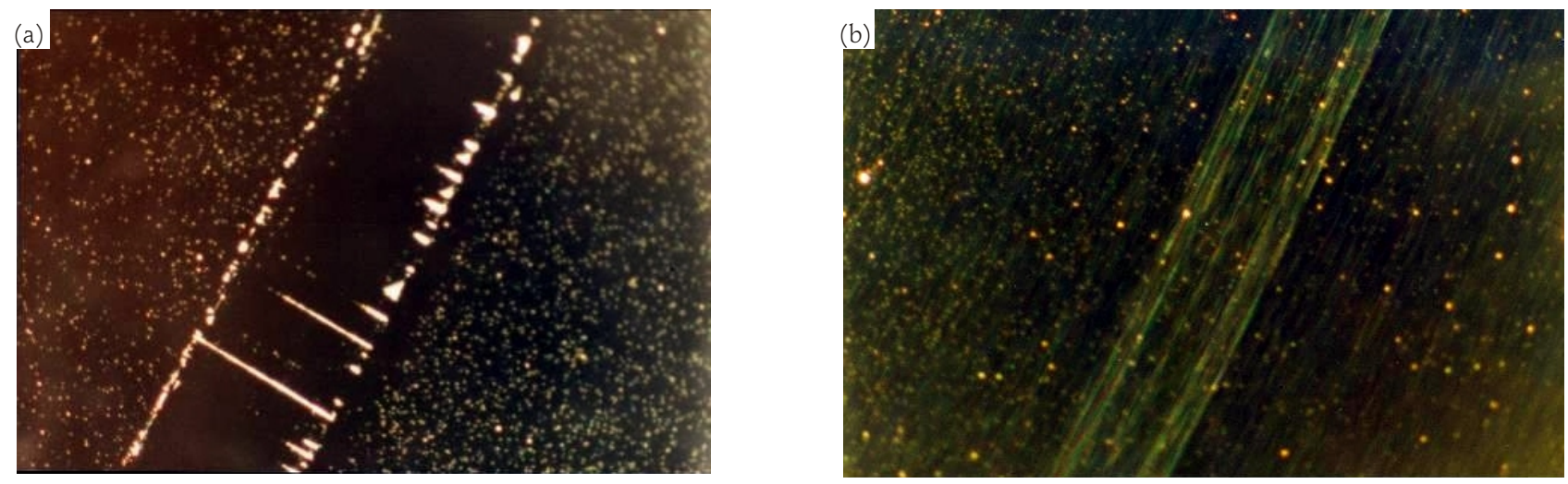

Figura 10. Imágenes correspondientes a superficie del disco a 32X. a) Se aprecian las marcas de vuelo sobre la superficie del disco, en una muestra que se ha sometido a condiciones de elevado nivel de humedad.

b) Se muestra evidencia del desgaste de la superficie del plato, por efecto del contacto con la cabeza lectora
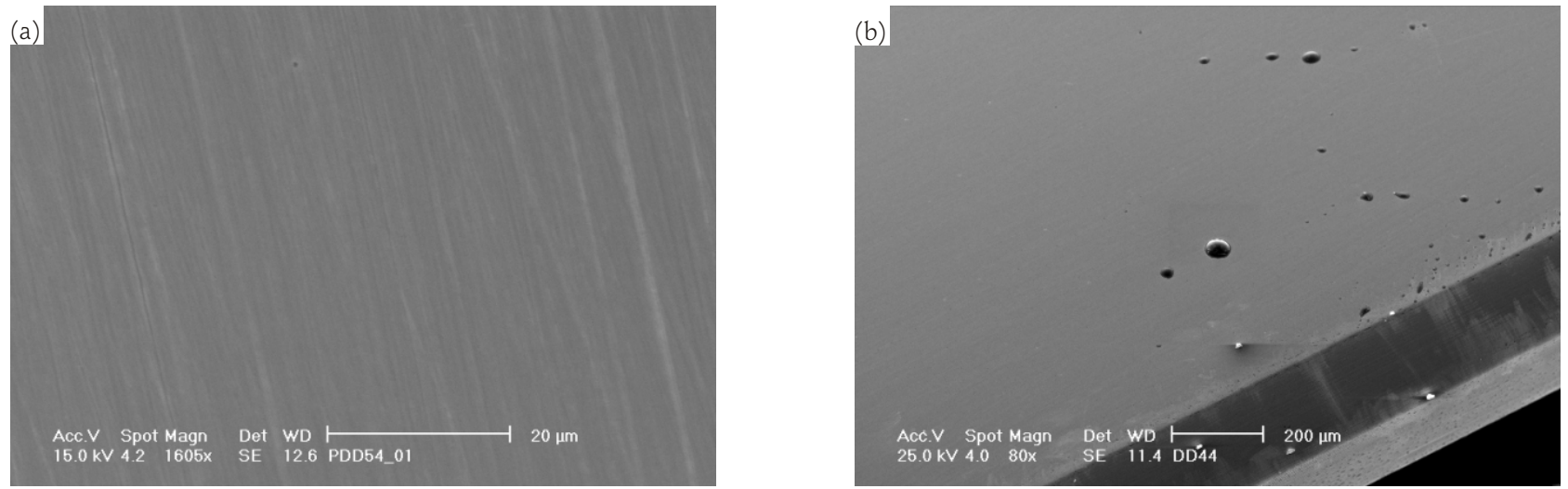

Figura 11. Imágenes de MEB. a) Apariencia de la superficie del plato mostrando marcas de vuelo, estando este dispositivo en condiciones de operación; b) Periferia del plato con presencia de discontinuidades

(a)

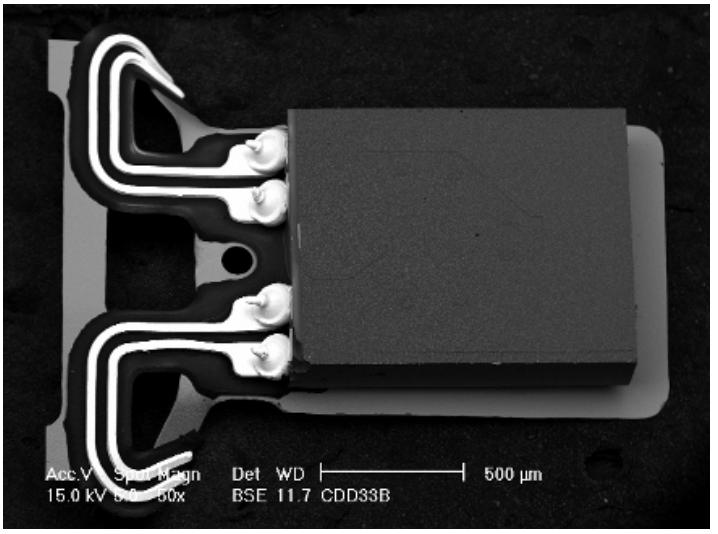

(b)

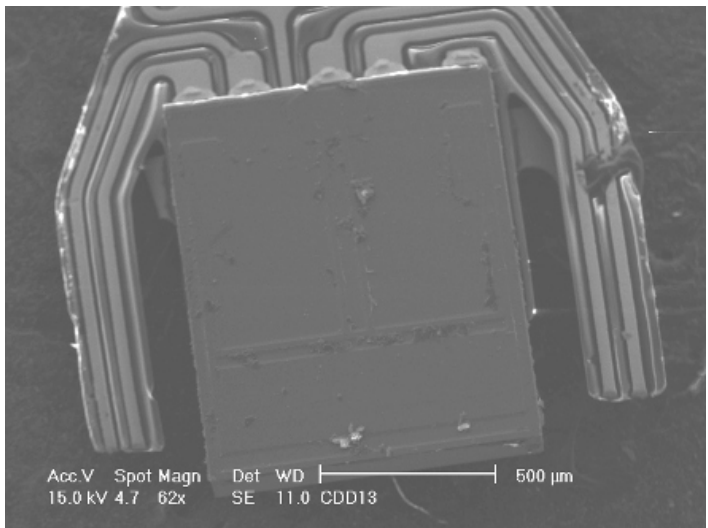

Figura 12. a) Cabeza típica de L/E nueva, sin evidencia de deterioro (conectores de oro);

b) Deterioro sobre la superficie de cabeza L/E ya en uso, lo cual propicia desprendimiento tanto del recubrimiento del plato como de la misma superficie de la cabeza producto de la colisión 
Las cabezas de L/E (figura 12), sufren también deterioro, particularmente desgaste y adhesión a la superficie del disco duro (figura 8) problemática que se manifiesta en la superficie de las cabezas de L/E, produciendo desprendimiento de material, lo cual provoca un efecto de sinergia.

\section{Deterioro por efectos mecánicos}

El deterioro por efectos mecánicos, tanto en la superficie del disco duro como de las cabezas de L/E, tiene su causa raíz en la distancia (tabla 1). Existe entre cabeza y superficie del plato, lo que incrementa la probabilidad de colisión con la superficie. La holgura o distancia de

Tabla 1. Tendencia en la miniaturización de la Cabeza de Lectura/ Escritura. El tipo de cabeza femto reduce las dimensiones lineales en un $30 \%$ y la masa en un $63 \%$, comparado a la cabeza de escritura pico

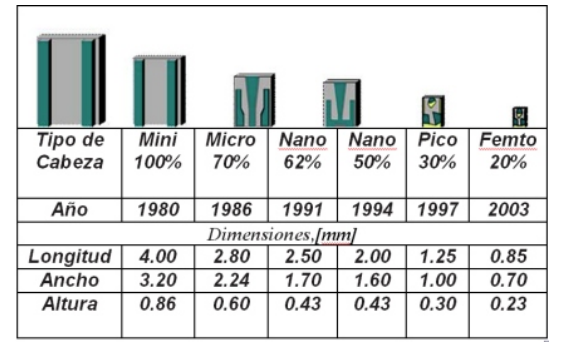

a)

\begin{tabular}{|c|c|}
\hline Material & Composición en masa \\
\hline Molibdeno & $79 \mathrm{Ni}$ \\
Permalloy & $17 \mathrm{Fe}$ \\
& $4 \mathrm{Mo}$ \\
\hline Mu-Metal & $77 \mathrm{Ni}$ \\
& $14 \mathrm{Fe}$ \\
& $5 \mathrm{Cu}$ \\
& $4 \mathrm{Mo}$ \\
\hline Hy-Mu & $70 \mathrm{Ni}$ \\
$800 \mathrm{~B}$ & $17 \mathrm{Fe}$ \\
& $4 \mathrm{Mo}$ \\
& $\mathrm{Nb}$ \\
& $\mathrm{Ti}^{\circ}$ \\
\hline Sendust & $85 \mathrm{Fe}$ \\
& $9 \mathrm{Si}$ \\
& $6 \mathrm{Al}$ \\
\hline Alfenol & $84 \mathrm{Fe}$ \\
& $16 \mathrm{Al}$ \\
\hline Intuctivo (MR) & $70 \mathrm{Al} \mathrm{O}_{3}$ \\
& $30 \mathrm{TiC}$ \\
\hline${ }^{\circ}$ Poca cantidad & \\
\hline
\end{tabular}

separación entre la cabeza de L/E y el plato, equivaldría en la actualidad a que un avión Boeing 747 volara a menos de un metro del suelo. La demanda de una mayor capacidad de almacenamiento de información y el consiguiente aumento en la densidad de datos se ha traducido en variaciones morfológicas de los sistemas, en el continuo refinamiento de los materiales (figura 13), así como a la mejora de los métodos de almacenamiento y al perfeccionamiento y optimización de los componentes electrónicos. Por consecuencia, cualquier anomalía en el funcionamiento, vibración, precipitación de condensados y presencia de partículas dará lugar a desgaste en la superficie del plato y deterioro de la propia cabeza de L/E.

El daño que cualquier contacto puede causar se determina al considerar las condiciones de operación de los platos (7200 a $15000 \mathrm{rpm}$, con una velocidad de 120 $\mathrm{Km} / \mathrm{h}$ en el borde). La característica principal de las cabezas lectoras es el sensor magnético y su superficie aerodinámica, la cual le permite planear sobre el disco a una distancia del orden aproximado de entre 10 a 25 nanómetros.

\section{Evaluación de la susceptibilidad de los SAMD al deterioro por efectos ambientales}

Una vez determinada que la causa fundamental del deterioro en el disco magnético es por fenómenos mecánicos se procedió a evaluar la susceptibilidad al daño por corrosión atmosférica de los diferentes elementos que constituyen el SAMD, para esto se empleó una cámara de atmósfera controlada (Cámara de Simulación Acelerada de Corrosión Atmosférica-CASACA-) de diseño propio (Cerrud et al., 2003).

b)

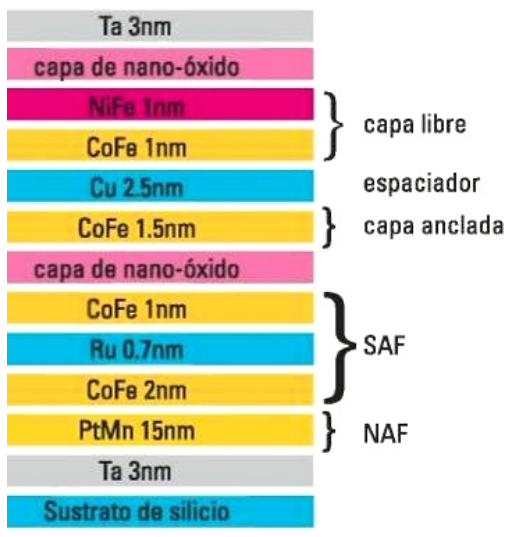

Figura 13. a) Materiales magnéticos utilizados en la fabricación de las cabezas de lectura/escritura. b) Sustratos que componen la cabeza de lectura - escritura 
Para la evaluación se utilizaron aquellos discos duros, cuyo diagnóstico indicó que se encontraban en condiciones de operación, las pruebas se realizaron en la CASACA programando ciclos de temperatura-tiempo de acuerdo a una señal trapezoidal, con un tiempo de ciclo de 20 minutos. El tamaño de muestra corresponde al $50 \%$ del total de unidades, provenientes de diversos fabricantes, esto bajo la presencia y ausencia de gases contaminantes.

Condiciones de ensayos acelerados por corrosión atmosférica:

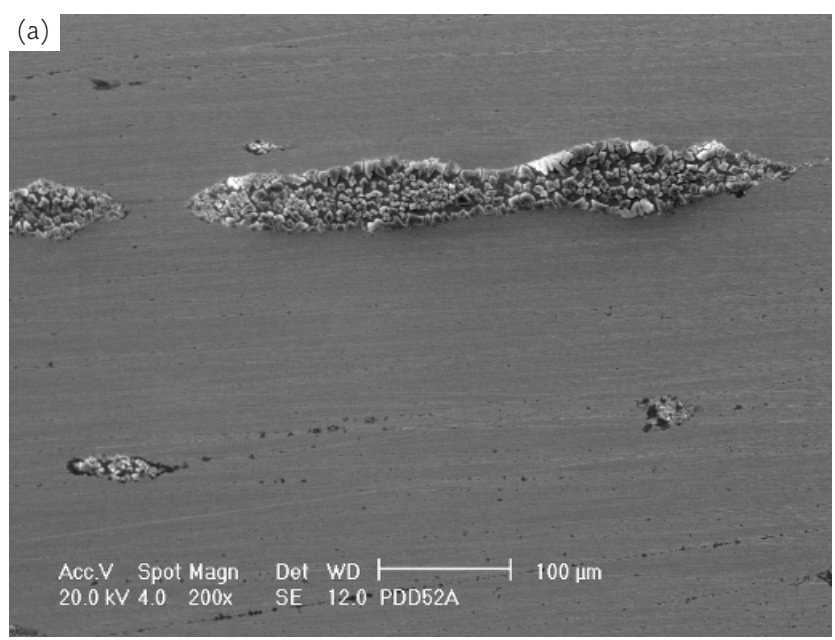

- Temperatura máxima $45^{\circ} \mathrm{C}$.

- Temperatura mínima $5^{\circ} \mathrm{C}$.

- \%Humedad Relativa Promedio 85\% a 90\%.

- Gases Inyectados: $\mathrm{SO}_{2}, \mathrm{NO}_{2}$.

La inspección con MEB muestra la formación de depósitos de dimensiones mínimas, en la superficie del disco y cabezas de L/E (figuras 14-17), esto para unidades que fueron sometidas a ensayos acelerados con elevados niveles de humedad, evidenciando que no se tiene hermeticidad al 100\% al interior del dispositivo.
Etiqueta A:

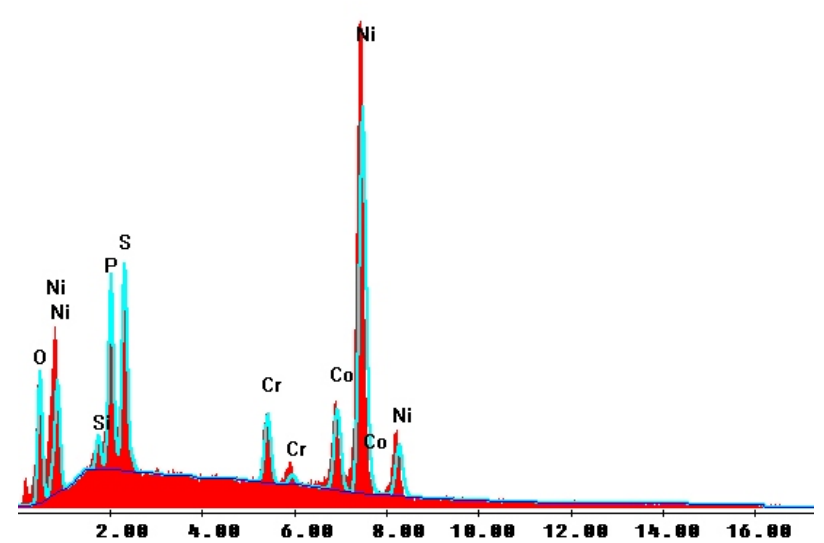

Figura 14 a). Formación de depósitos de corrosión en la superficie del plato, debido al desprendimiento de la capa de carbono producto del contacto existente entre cabezal y plato. El fenómeno inicial es de tipo mecánico (200X). b) El espectro muestra dentro de los elementos cuantificados la presencia de azufre y oxigeno como agentes ambientales que propician la corrosión

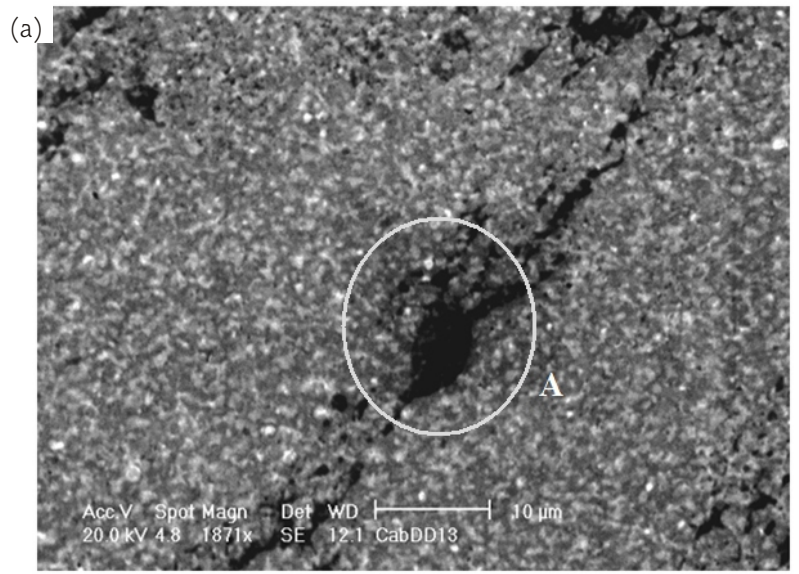

(b)

Etiqueta A:

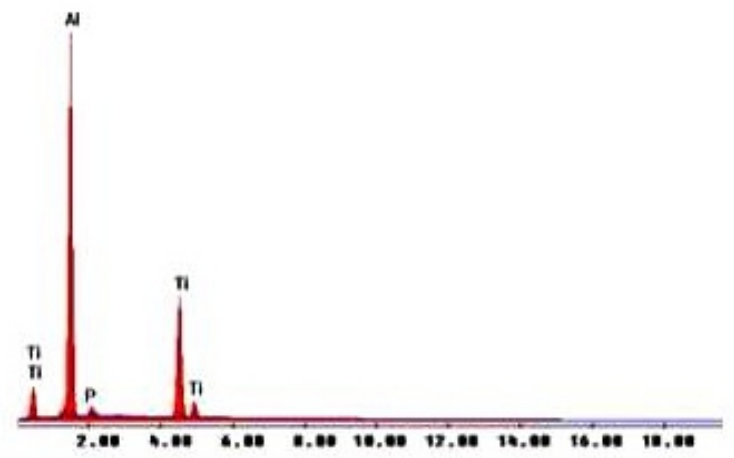

Figura 15. a) Esta imagen muestra la superficie de la cabeza de $L / E$, donde se aprecia claramente la pérdida de material acompañado de una grieta en la zona seleccionada "A". b) Se muestra el espectro del análisis y cuantificación de los materiales presentes en " $A$ ", donde no existe evidencia de elementos que propicien la corrosión 

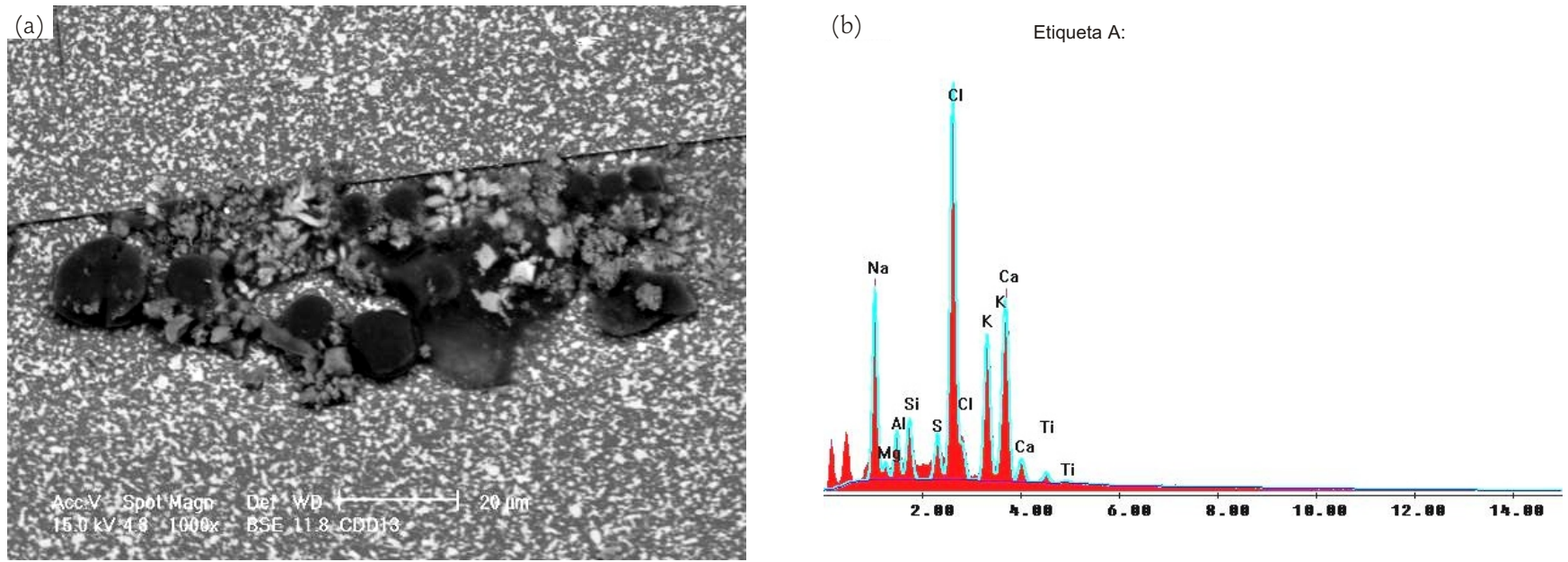

Figura 16. a) Formación de depósitos sobre la superficie de la cabeza de $L / E$;

b) En el espectro mostrado es notoria la presencia de $\mathrm{Cl}$, y en menor porcentaje de $\mathrm{S}$. La muestra proviene de la costa, durante el ensayo acelerado no se utilizó cloro, por lo que se concluye que el depósito observado existía antes de la prueba acelerada pero no fue suficientemente grave para causar la falla del disco
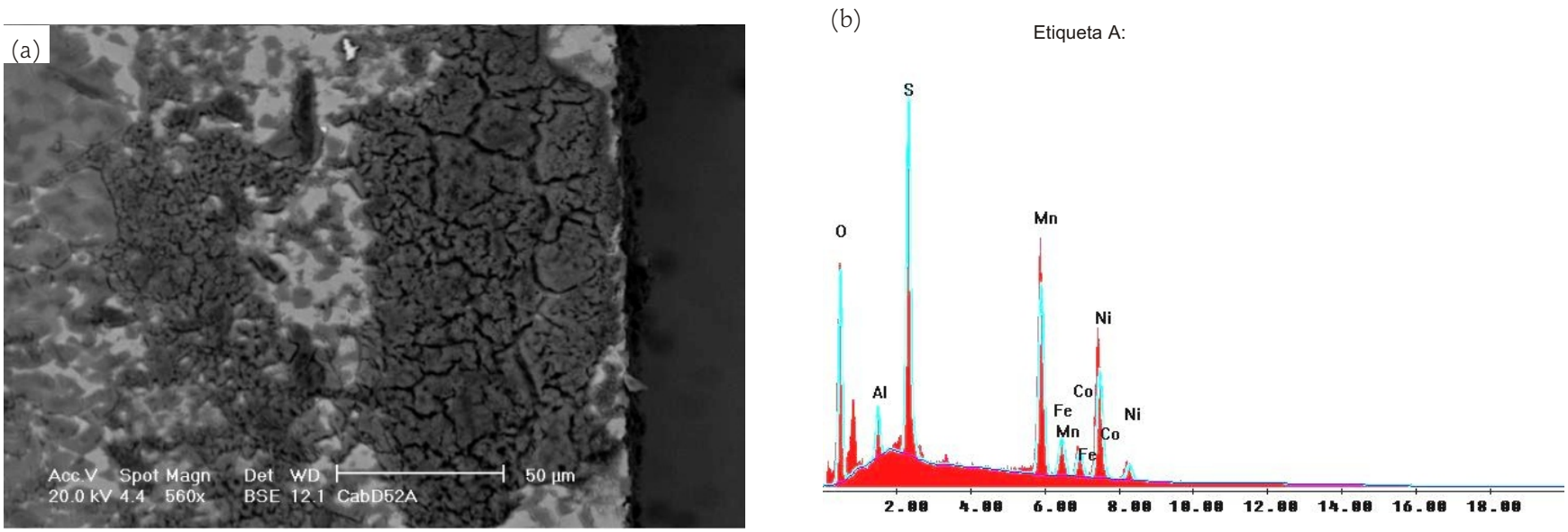

Figura 17. a) Evidencia de la presencia de material producto de la acumulación de condensado justo en los bordes de la cabeza $L / E, b)$ El espectro muestra la presencia de azufre y oxigeno justo en la zona mencionada

\section{Resultados}

En términos generales, al realizar la inspección y evaluación de las muestras que fueron sometidas a los ensayos acelerados sólo se presentan evidencias mínimas de formación de depósitos de corrosión en la superficie del plato de algunos de los especímenes, esto refiere a que no se ha garantizado la hermeticidad del disco. Los depósitos principales se presentan en pines de conexión, situación que en algunos casos genera inconvenientes y hasta la falla aparente del disco; sin embargo, después de la limpieza de éstos el SAMD continuó operando con normalidad.

En todos los casos, los depósitos observados se correlacionan con efectos mecánicos previos producto de la colisión entre el cabezal y la superficie del plato. 
(a)

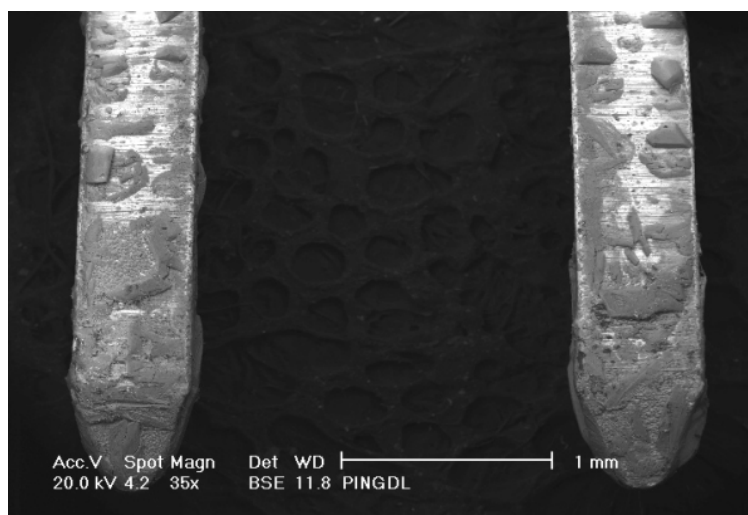

(b)

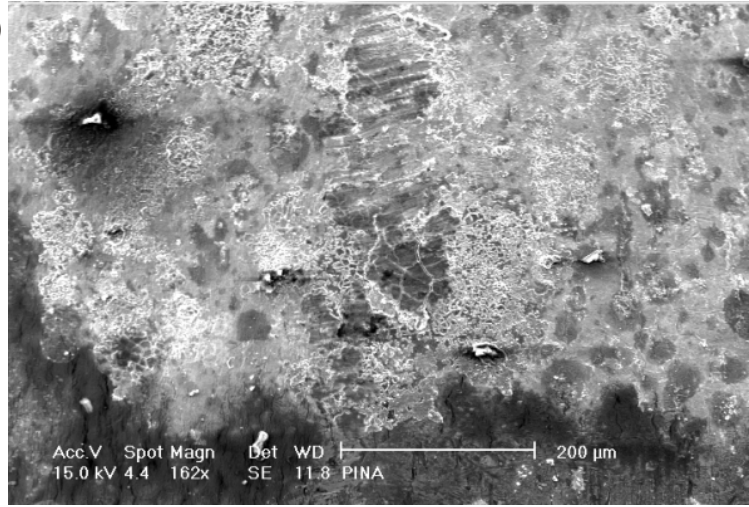

Figura 18. a) Pines IDE con acumulación de sulfatos, debido a la exposición de gases en ensayos acelerados de corrosión. b) Pines de alimentación de energía mostrando cambios de tonalidad y presencia de depósitos de corrosión

Las observaciones realizadas indican una elevada frecuencia en deterioro por fenómenos corrosivos en componentes electrónicos de la tarjeta controladora del SAMD, así como en pines IDE y de alimentación, los cuales se evaluaron mediante MEB (figura 18).

Deterioro por corrosión atmosférica en componentes electrónicos de SAMD

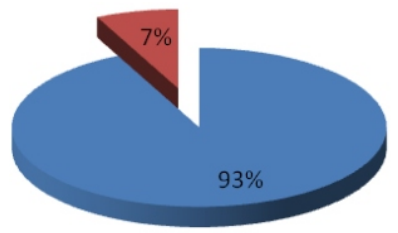

- Evidencia de Corrosión

- Sin evidencia de Corrosión

Figura 19. Frecuencia con la que se observa deterioro por corrosión en los componentes electrónicos del SAMD al ser evaluados mediante el protocolo de simulación acelerada

Resultado de la Inspección Interna del SAMD al término de la Prueba 2
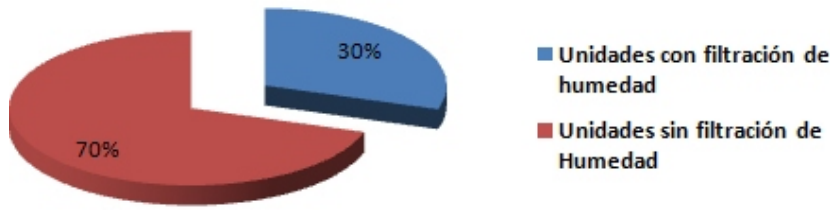

Figura 20. La tendencia indica que una de cada tres unidades tiende a presentar filtración de agentes externos al interior del $S A M D$, por ende, se eleva la probabilidad de tener desgaste en la superficie magnética
Las muestras sometidas a ensayos cíclicos de humedad-temperatura (en ausencia de gases contaminantes) en la CASACA, indican que no existe evidencia de falla por corrosión en el plato, esto a pesar de la presencia de un elevado nivel de humedad (figuras 19 - 20), el cual se constata por las marcas de condensado observadas.

Sin embargo, los componentes electrónicos, así como pistas impresas de la tarjeta controladora del SAMD muestran notable deterioro y una clara evidencia de corrosión con formación de depósitos (figura 21), siendo esto un factor determinante para el mal funcionamiento del dispositivo.

Cuantificación de unidades con indicios de herrumbre en pines

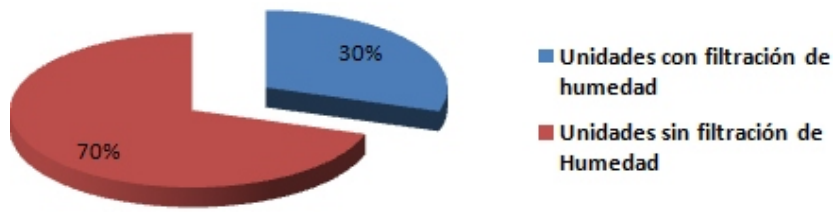

Figura 21. La humedad es factor fundamental para el deterioro por $C A$, lo anterior, sin considerar el efecto de los gases contaminantes

\section{Conclusiones}

Si bien, la superficie de almacenamiento magnético de datos es susceptible de presentar deterioro por corrosión, esto en virtud de su composición y de la factibilidad de que se genere una celda galvánica, la superficie del disco es el elemento menos susceptible a este tipo de fenómenos, 
DOI: http://dx.doi.org/10.22201/fi.25940732e.2010.11n4.037

Ortiz-Prado A., Schouwenaars-Franssens R., Jacobo-Armendáriz V.H. y Sánchez-Pérez F.

aún en el caso de condiciones extraordinariamente agresivas, desde el punto de vista de la combinación de un medio ambiente agresivo (ensayos acelerados) con una falta de hermeticidad del SAMD (diseño de la carcasa).

Sin embargo, pueden presentarse inconvenientes por partículas que se desprenden de otros elementos y se depositan sobre la superficie de almacenamiento magnético de datos.

Estas partículas pueden provenir del medio ambiente, de la interferencia entre disco y cabeza lectora o de otros elementos que sí presentan deterioro por corrosión. Las pruebas de corrosión llevadas a efecto en la cámara de simulación acelerada demuestran que una elevada humedad relativa, es precursora de este tipo de fenómenos, los cuales sí causan falla en el SAMD, pero debida ésta a la formación de depósitos en los pines de conexión o en los elementos de la tarjeta controladora. En este caso, queda demostrado también que la falta de actividad del disco (paro por periodos prolongados) bajo condiciones de elevada humedad relativa, da lugar a su falla por efecto de la formación de depósitos entre el cabezal de lectura y el disco.

Por consecuencia, se concluye que la baja humedad relativa característica de ciudades altamente contaminadas como Monterrey, Guadalajara y la propia Ciudad de México, no representan un factor fundamental de falla, y en consecuencia, se caracterizan por un reducido índice de deterioro en el equipo electrónico, para esto habrá que considerar la existencia de microclimas en dichas zonas metropolitanas, así como de las condiciones particulares de operación de dichos equipos.

En los especímenes que fueron sometidos a pruebas aceleradas de deterioro por corrosión atmosférica se ha detectado que se presenta sobrecalentamiento en ciertos circuitos integrados, lo cual se manifiesta como una reducción en la velocidad de respuesta de la computadora independientemente del resultado del diagnóstico. Las observaciones realizadas permiten concluir que a un número reducido de ciclos de temperatura-humedad se presenta acumulación de condensado sobre las tarjetas controladoras, propiciando la formación de depósitos, principalmente en terminales de los circuitos integrados, en capacitores, así como en terminales de los pines IDE que se encuentran en las mismas.

En términos generales, se puede decir que el mal funcionamiento de un SAMD se da, de acuerdo a sus componentes, en el siguiente orden de deterioro:

1. Pines IDE - Alimentación. Elemento principal que sufre deterioro provocando una mala transmisión de la señal.
2. Circuitos integrados. Se presenta sobrecalentamiento al momento del diagnóstico, llevando al calentamiento de la computadora.

3. Pistas conductoras de la tarjeta impresa. Se presenta deterioro por causa de acumulación de condensado, son muy susceptibles a la presencia de humedad.

4. Capacitores y demás elementos. Sufren deterioro con menor efecto.

5. Sello protector de la carcasa. Aunque se han mejorado los materiales de este elemento, sigue existiendo filtración de humedad en el SAMD.

6. Cabeza de L/E. El deterioro sufrido en estos elementos se da por el choque entre cabezal y plato, debido a la vibración existente o producto de la interrupción en el envió de la señal para su óptimo funcionamiento. También pueden presentar inconvenientes luego de paros muy prolongados en presencia de elevada humedad relativa combinada con fallas de hermeticidad de la carcasa.

De acuerdo a la hipótesis planteada, se concluye que la corrosión en el plato del SAMD no es factor determinante en el mal funcionamiento del dispositivo, todo recae en el efecto corrosivo existente en los componentes electrónicos de la tarjeta controladora del dis$\mathrm{co}$, por consiguiente, la susceptibilidad de deterioro por condiciones ambientales de los SAMD es similar a la de cualquier dispositivo electrónico y no deberá ser agrupado como un tipo particular de fenómeno, ya que los factores y condiciones de daño coinciden con lo reportado en circuitos impresos (Ortiz, 2004).

\section{Agradecimiento}

Se brinda especial agradecimiento a la Dirección General de Apoyo a Personal Académico (DGAPA), por los recusos brindados mediante el proyecto PAPIIT-IN 102706 "Deterioro por efectos ambientales en sistemas de almacenamiento magnético de datos". Así como al Ing. Álvaro Contreras García, M en I. Ignacio Cueva Güitrón y técnico académico Germán Álvarez Lozano.

\section{Referencias}

Barrios L, Sánchez F. Deterioro por corrosión atmosférica en sistemas de almacenamiento magnético de datos. Tesis (Licenciatura). México DF. Facultad de Ingeniería, Universidad Nacional Autónoma de México. 2005.

Cerrud S., Ortiz A., Jacobo V., Schouwenaars R. Design, Construction and Testing of a System for Accelerated Simulation of Damage by Atmospheric Corrosion on 
DOI: http://dx.doi.org/10.22201/fi.25940732e.2010.11n4.037

Análisis de las causas de deterioro en sistemas de almacenamiento magnético de datos

Electronics Equipment. Corrosion Reviews, Vol. 21, 2003.

Gouda V.K., Carew J.A., Riad W.T. Investigation of Computer Hardware Failure due to Corrosion, British Corrosion Journal, Vol.24, 1989.

Goglia P., Berkowitz J. Diamond Like Carbon Applications in High Density Hard Disk Recording Heads. Diamond and Related Materials, 2001.

Guttenplan, J.D. Corrosion in the Electronics Industry. Metals Handbook. ASM International. Vol.13. 1987.

Leygraf C.D., Graedel T. Atmospheric Corrosion. New York. John Wiley \& Sons. 2000.

Marcus, P. Corrosion Mechanisms in Theory and Practice. Marcel Dekker Publisher. 2002.
Ortiz A. Simulación acelerada de la corrosión atmosférica en equipo electrónico. Tesis (Doctoral). México DF. Facultad de Ingeniería, Universidad Nacional Autónoma de México. Posgrado de Ingeniería. 2004.

Ortiz A. et al. Análisis del deterioro que por corrosión atmosférica se presenta en sistemas de almacenamiento magnético de datos. Memorias del XI Congreso Internacional de la SOMIM, 2005.

Ortiz A. et al. Estudio del deterioro por corrosión atmosférica en discos duros y cabezas de Lectura/Escritura. Memorias del XIII Congreso Internacional de la SOMIM, 2007.

White E., Slensky G. Case Histories and Failures of Electronics and Communications Equipment. Metals Handbook, ASM, 1987. 
DOI: http://dx.doi.org/10.22201/fi.25940732e.2010.11n4.037

Ortiz-Prado A., Schouwenaars-Franssens R., Jacobo-Armendáriz V.H. y Sánchez-Pérez F.

\section{Semblanza de los autores}

Armando Ortiz-Prado. Se graduó como ingeniero mecánico electricista (Ing. mecánica) por la Facultad de Ingeniería de la UNAM en 1980, posteriormente obtuvo la maestría y el doctorado en ingeniería mecánica. Es profesor desde hace 29 años, impartiendo cátedra en las áreas de procesos de manufactura y ciencia de materiales. Actualmente es profesor titular "C" de tiempo completo definitivo y tutor doctoral de los posgrados de ciencia e ingeniería de materiales y de ingeniería. Sus líneas de investigación son modelado de procesos de conformado mecánico, análisis de falla, comportamiento mecánico de biomateriales y corrosión en equipo electrónico. Ha desarrollado proyectos y estudios para diferentes empresas privadas y entidades gubernamentales, orientados éstos al desarrollo y mejora de métodos de manufactura, desarrollo de materiales, corrosión y análisis de falla; lo cual se traduce en más de 50 estudios y asesorías para la industria, 12 proyectos de investigación y desarrollo tecnológico. A la fecha, suma más de 90 tesis de licenciatura y se encuentra dirigiendo cuatro tesis doctorales. Su experiencia se ha transmitido a través de 7 textos para licenciatura en las áreas de manufactura, materiales y corrosión. Asimismo, su productividad incluye más de 15 artículos en congresos internacionales, más de 80 artículos en congresos nacionales y más de 20 artículos en revistas arbitradas. Fue galardonado por la UNAM con la Distinción Universidad Nacional para Jóvenes Académicos (Docencia en ciencias exactas) en 1989. Su productividad académica y científica ha sido reconocida por la UNAM, a través del otorgamiento del nivel D del PRIDE y por el CONACYT con el nombramiento de Investigador Nacional Nivel I.

Rafael Schouwenaars-Franssens. Obtuvo el grado de maestro en ingeniería de minas en la Universidad de Lovaina, Bélgica y el doctorado en ingeniería mecánica en la FI-UNAM. Ocupa el nivel I en el sistema nacional de investigadores. Durante cuatro años estuvo activo como investigador trabajando en proyectos industriales relacionados con la textura cristalográfica en aceros en el Departamento de Metalurgía e Ingeniería de Materiales de la misma Universidad. Su trabajo en México lo inició como experto en microscopía electrónica dentro de la Unidad de Investigación y Asistencia Tecnológica en Materiales (UDIATEM). Posteriormente, combinó estas actividades con una carrera de profesor en la Facultad de Ingeniería. En el ámbito tecnológico se dedica al análisis de falla y la optimización de procesos en la industria metal-mecánica, con un enfoque especial hacia las aleaciones tribológicas dúctiles. Esto ha llevado a una serie de estudios científicos relacionados con las propiedades mecánicas de materiales complejos, los cuales se han publicado en 25 artículos internacionales, 7 en revista nacional y 37 que se han presentados en 30 congresos tanto internacionales como nacionales. Es árbitro de varias revistas nacionales e internacionales y es miembro del comité científico de dos series de congresos internacionales de primer nivel.

Víctor Hugo Jacobo-Armendáriz. Ingeniero mecánico electricista (área de Ing. Industrial) por la Facultad de Ingeniería de la Universidad Nacional Autónoma de México (UNAM), en donde también obtuvo los grados de maestro y doctor en ingeniería. Actualmente funge como profesor titular "B" de tiempo completo definitivo en el área de manufactura y materiales, en la propia FI-UNAM. Su línea se ha orientado al análisis de falla, ciencia de materiales y sistemas expertos. Ha participado en diversos proyectos y estudios al desarrollo y mejora de métodos de manufactura, corrosión y análisis de falla. Ha dirigido más de 10 tesis de licenciatura y publicado 2 textos (análisis de falla, corrosión y protección), más de 10 artículos en congresos internacionales, más de 30 en congresos nacionales y más de 10 en revistas arbitradas. Actualmente es miembro del sistema nacional de investigadores (NIVEL I) del CONACYT.

Francisco Sánchez-Pérez. Ingeniero mecánico egresado de la Facultad de Ingeniería de la UNAM en 2005, cuenta con estudios de maestría en ciencias e ingeniería de materiales dentro del Instituto de Investigaciones en Materiales de la UNAM. Es profesor de asignatura de la Facultad de Ingeniería. Pertenece al grupo de investigación de la Unidad de Asistencia Técnica en Materiales de la UNAM como colaborador dentro de proyectos enfocados al área de manufactura y materiales desde el 2003. Participa desde hace tiempo en proyectos relacionados al área de corrosión atmosférica en equipo electrónico. 